\title{
An Electronic Information System to Facilitate Parking at the Prophet's Mosque in Medina
}

\author{
Ahmad Abdelqader Abuseenia, Husam Omar Alfahilb \\ Department of MIS \\ Taibah University \\ Medina, Saudi Arabia \\ aseeni@taibahu.edu.sa,hfahl@taibahu.edu.sa
}

\begin{abstract}
In light of today's rapid technological development, it often necessary to use internet services to plan daily life activities. For example, many people depend on these services to determine the dates of their travel and the best and shortest route to follow. In this study, an electronic information system is proposed that will provide information about the availability of parking spaces at the Prophet's Mosque in Medina, as well as assistance in finding the shortest route to the open parking spaces. The system helps visitors either through a mobile application or-for users who do not use mobile applicationsthrough information panels at the main gates and the entrance to each parking lot.
\end{abstract}

Keywords: Prophet’s Mosque, JavaScript Object Notation (JSON), Prophet's Mosque Parking

Received: 2 May 2018, Revised 5 June 2018, Accepted 20 June 2018

DOI: $10.6025 /$ jism/2018/8/3/105-109

(C) 2018 DLINE. All Rights Reserved

\section{Introduction}

The Prophet's Mosque in Medina is visited by millions of people each year. The mosque's parking lots can accommodate 4300 cars distributed over 24 units, including 11 units on the upper floor and 13 units on the lower floor. Twelve of these units are allocated for annual and monthly subscriptions, and 12 are allocated for cash parking. Each parking unit is monitored by a device that controls entry and exit, [5]. There are no panels at the unit entrances to indicate the number of spaces available, and there are no similar panels at the main entrances. Thus, a visitor can enter the parking lot without knowing the number of available spaces, the location of available spaces, or the shortest route to the nearest available spaces.

In this study, an electronic information system is proposed to facilitate the communication of parking availability to visitors in two ways. In the first, which is suitable for visitors who use smartphones, the information is transmitted via an application connected to the Haram parking management system. In the second, information is advertised on large, customized boards placed at the main entrance of the parking lot and the entrances of each internal unit. 
The research problem and objectives will be described in Sections 2 and 3. Section 4 presents a survey of previous studies, and the details of the new system are presented and explained in Section 5. The results and advantages of the proposed system will be detailed in Section 6.

\section{Research Problem}

Currently, when a person wants to visit the Prophet's Mosque, he has no way of knowing the availability of vacant spaces in the mosque parking lot. Thus, he is forced to enter the parking area and search for a space without the help of explanatory panels indicating the number of vacant spaces in each parking unit.

\section{Research Objectives}

The aim of this study was to present an electronic information system that enables the user to find information about vacant parking spaces at the Prophet's Mosque. This system is connected to a mobile application with a GPS system in order to guide the user to the nearest vacant space. The system also provides guidance panels at the main entrance gates and the entrance to each parking unit, so that users who do not use mobile applications can also access the required information.

\section{Background Information}

As noted in [1], it can be quite difficult to find an empty parking space, at most local parking lots, drivers need to find empty parking spaces themselves, and they can waste a lot of time searching for an empty space if they do not know where the spaces are located, especially when there are only a few empty spaces available in each row of the parking lot. Therefore, it is important to have an effective tracking system that can display the empty parking available in each row and guide the driver to it. This project prototype can act as a Wayfinder to guide drivers in the parking lot to an available parking space. The proposed system represents a fully automated tool that requires minimal human intervention and overcomes the limitations of existing systems.

In [2], the authors describe the use of a parking system that assists drivers in an efficient manner from their entrance into a parking lot until their finding of a vacant parking space. The prototype system uses an Android operating system to effectively guide drivers; it is designed to encompass two floors with three parking spaces on each floor. The system architecture defines the essential design features such as the locations of sensors, the required number of sensors, and the Liquid Crystal Display (LCD) display board.

In [3], the authors say that all around the world, parking spaces have been constructed with access control points in place. For example, at shopping malls and airports, some control points are automated so that users can use self-service parking, while others are manned by control personnel.

The authors in [4] confirmed that there is a need to create and design a parking management system using a mobile application. They tested an application that can interact with sensors, enabling drivers to locate empty parking spaces and determining the place where a client has parked his car. The resulting system organizes the parking process and makes it easier. Using a custommade sensor based on a TCRT5000 photo transistor with an infrared transmitter, the system is capable of determining the presence or absence of a car in the lot. The sensor status is sent to a database using an Arduino Mega 2560 microcontroller.

\section{The Current System}

The system currently used in each internal parking unit at the Prophet's Mosque involves an electronic input/output barrier that opens and closes based on the number of cars entering and exiting the unit; it subtracts from the total number of parking spaces to output the number of available spaces. However, the system does not link to an external application, and there are no billboards at the entrances of the internal units or at the main entrance. In addition, the method used to determine the number of available spaces is not accurate, especially when certain cars take up more than one parking space.

\section{Methodology}

First, a custom-made sensor based on a photo transistor with an infrared transmitter was produced. This system is capable of determining the presence or absence of a car in the lot, based on the system referred to [4]. Second, the sensor status is sent to

$106 \quad$ Journal of Information \& Systems Management Volume 8 Number 3 September 2018


the access point at each internal parking unit and then to a database that maps to the reporting system. Third, the reporting system sends information about the presence or absence of a car in the lot to the Prophet's Mosque parking mobile application and LCD displays, as illustrated in Figure 1. Fourth, the mobile application must be capable of sending and receiving data from the database; we suggest using JavaScript object notation (JSON) format, as has been advised in a previous study about the effectiveness of this type of system [4].

Using the mobile application, the customer can locate available parking spaces; moreover, as soon as the visitor enters the Prophet's Mosque parking area, a timer starts calculating the time spent there, and based on this time interval, the client is notified of the parking fee withdrawn from his account.

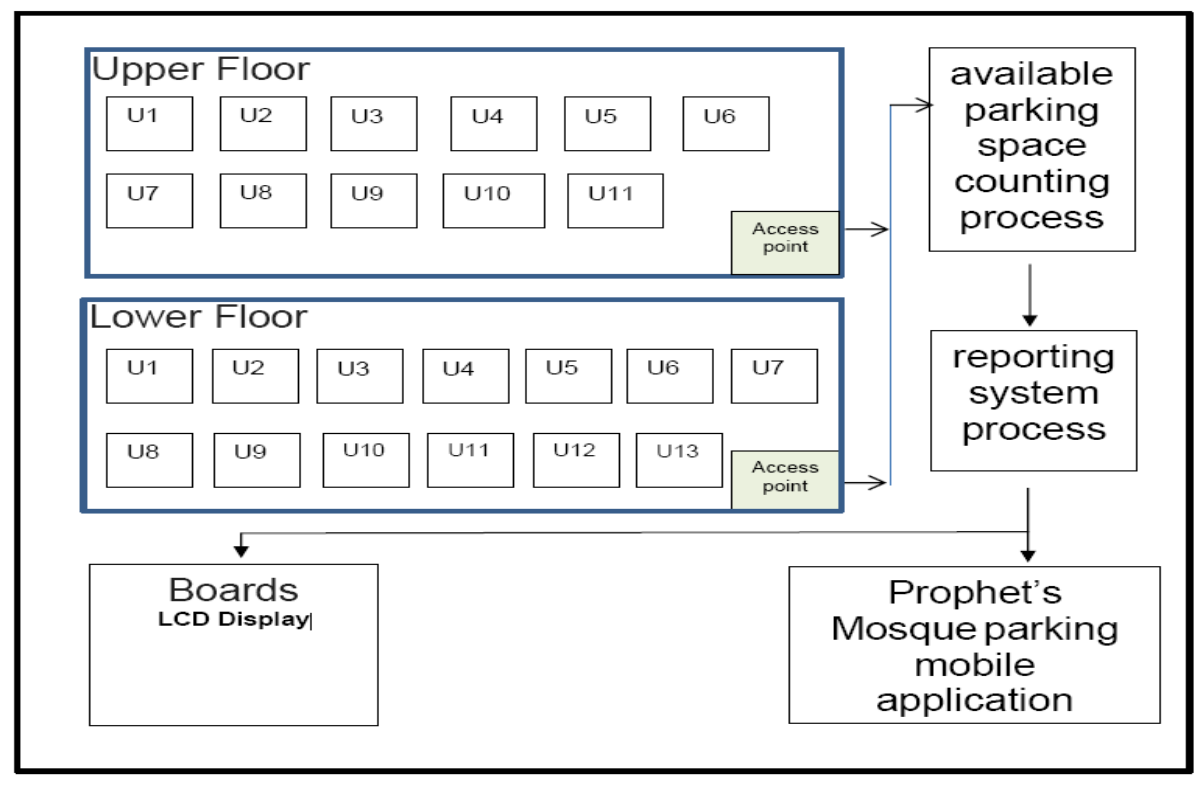

Figure 1. Prophet's Mosque Parking System Methodology. ("U” means internal unit in prophet’s mosque parking)

Available Parking Space Counting Process: This process is relayed to a database system that collects and arranges information about the number of vacant parking spaces for each of the 24 units of the Prophet's Mosque parking system, as illustrated in Table 1.

\begin{tabular}{|c|c|c|}
\hline Floor number & Unit number & Number of available spaces \\
\hline Upper Floor & $1-11$ & 156 \\
\hline Lower Floor & $1-13$ & 23 \\
\hline
\end{tabular}

Table 1. Vacant parking space report

Reporting System Process: This process initiates reports as required from the Prophet's Mosque parking mobile application and parking management system.

Boards (LCD displays): LCD displays or lighted boards will be placed at the main entrance gate and at the entry of each unit; they will display the number of vacant parking spaces in the parking area, as shown in Figures 2 and 3.

Prophet's Mosque Parking Mobile Application: This is a mobile application that eases drivers' search for a parking space at the Prophet's Mosque parking area. They can find and book parking with just a few taps on-the-go instead of circling the block looking for parking, as shown in Figure 4. 

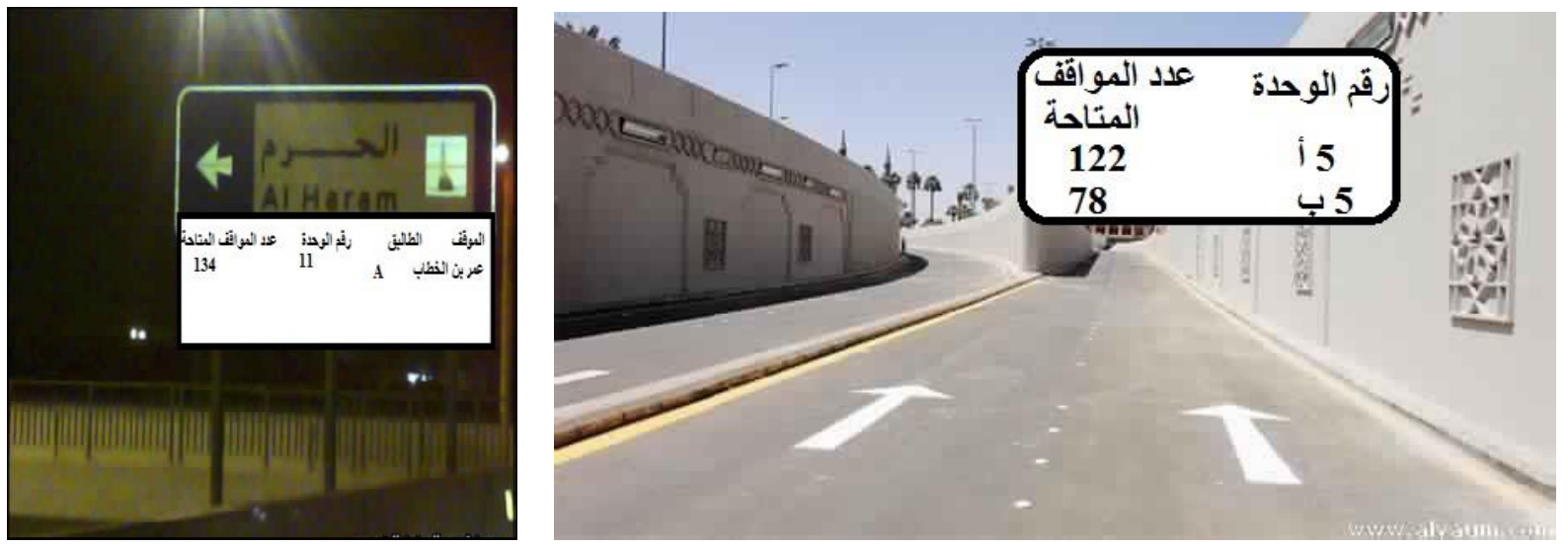

Figure 2. LCD Display At The Main Gate

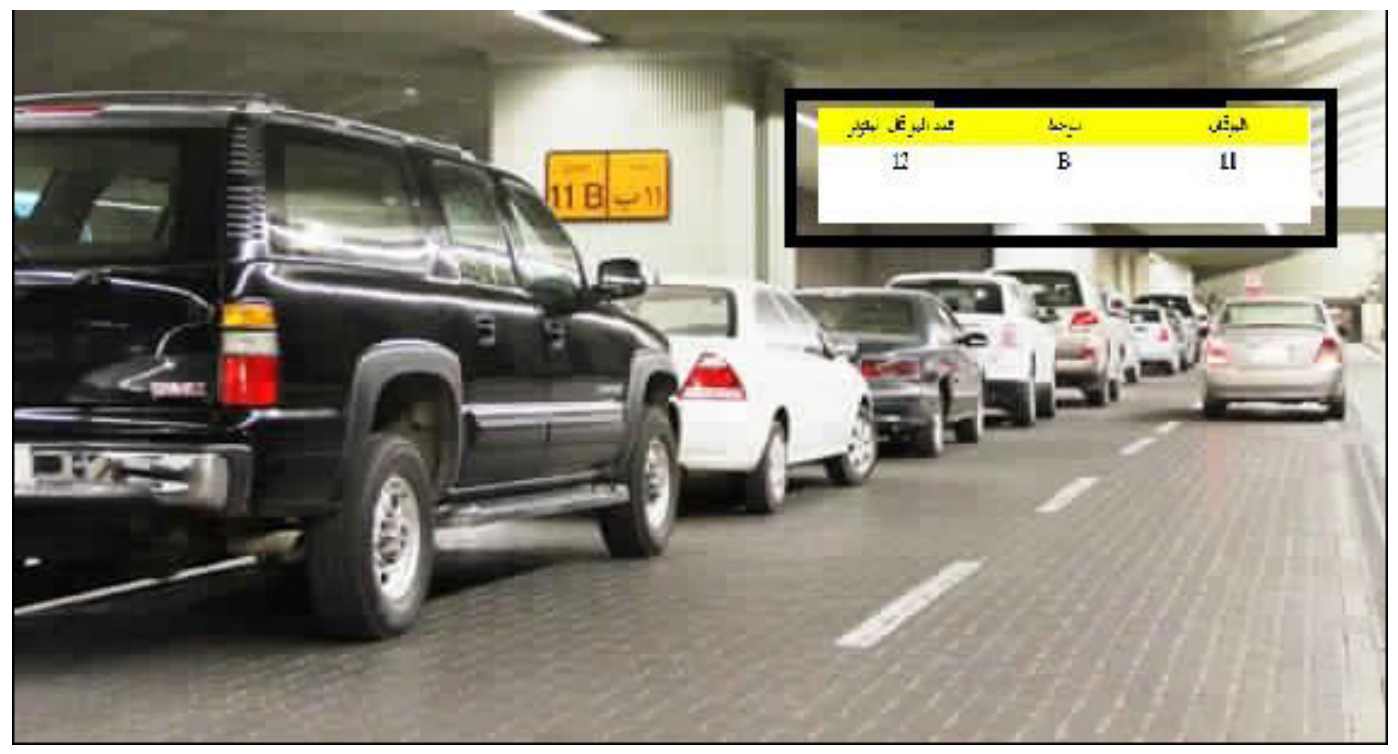

Figure 3. LCD Displays at the Internal Unit Gates

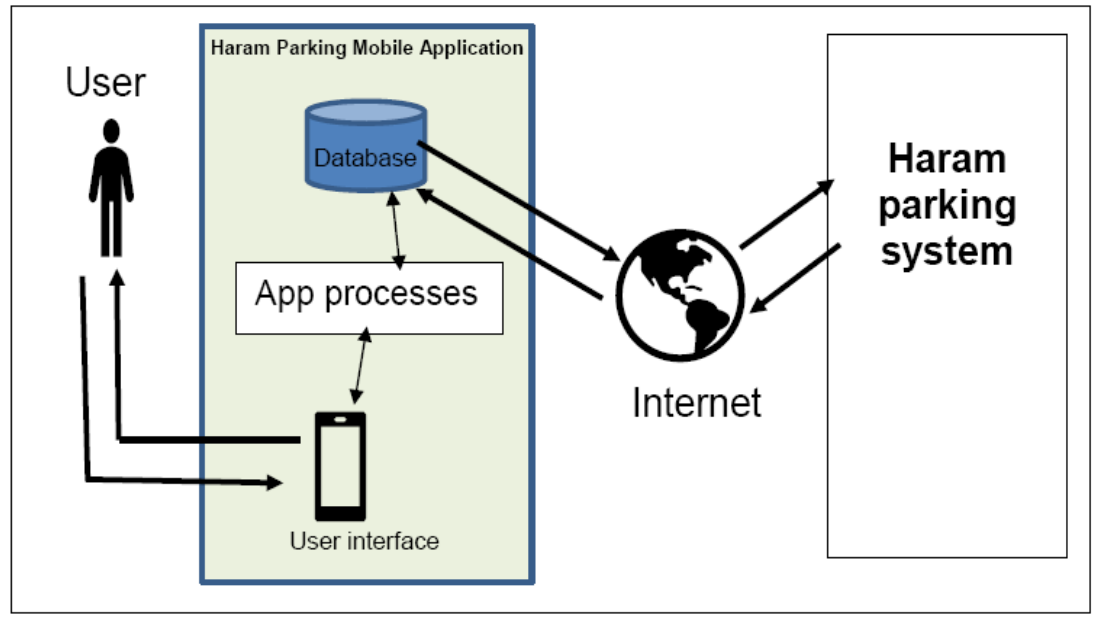

Figure 4. Prophet's Mosque Parking Mobile Application 


\section{Conclusion}

The proposed system will effectively facilitate the process of finding available parking in the Prophet's Mosque parking area. The advantage of the system is that it will provide information in different ways depending on a visitor's needs. The mobile application can be used to identify available parking before a visitor goes to the mosque, and he will be guided to the parking space by the navigation system. The system also provides information to visitors who do not use mobile applications through guide boards placed at the main entrance and subsidiary internal units that display information about the available parking. The system also facilitates payment for parking through an electronic payment system linked to the mobile application. This will reduce the need for exact currency or change upon exiting.

\section{Advantages of the proposed system:}

1. The visitor will be able to know the status of available parking before starting his trip.

2. Payment for parking will be easier because it can be done electronically, avoiding the need for a queue at checkout.

3. The problem of congestion at the main entrances of the Prophet's Mosque parking area will be solved because the boards at these entrances will inform visitors about the number of available parking spaces. The drivers will not enter the parking areas unless there are available parking spaces.

4. Crowding in a long queue at the entrance of each parking unit inside the Prophet's Mosque parking area will be avoided because the boards placed at the entrances will inform the visitors about the number of available parking spaces. Thus, the drivers will not wait in the queue unless there are available spaces.

5. The process of finding the desired parking space will be resolved because the application will help the visitor navigate to the correct location.

\section{References}

[1] Ketan, S., Gaikwad, S., Kedari, U., Bhokre, D., Bonde, J., Rohit, S. (2014). Automated car parking system commanded by Android application. Intl. J. Computer Sci. Inform. Tech., 5 (3), 3001-3004.

[2] Nikhil, P., Chhaya, N., Sunita, K. (2016). Car parking system: An Android approach. Intl. J. Innovative Res. Computer Comm. Engin., 4 (3).

[3] Anthony, M. (2009). The design of an intelligent parking system using wireless sensor networks and multi-protocol label switching. M.S. thesis submitted to the Department Of Computer Science, Faculty of Science, University of Cape Town.

[4] Michel, O., Bahaa, S., Elio, S., Amer, M. (2015). Parking management system using a mobile application. 21ème Conférence Scientifique Internationale du LAAS April 21, 753-758.

[5] The Prophets Mosque, General Presidency of the Grand Mosque \& the Prophets Mosque, 9:30 am, 25-12-2017, https:// www.gph.gov.sa/ar-sa/Pages/default.aspx. 